\title{
Circulating sex-steroids and Staphylococcus aureus nasal carriage in a general female population
}

\author{
Dina B Stensen 1,2, Lars Småbrekke ${ }^{3}$, Karina Olsen ${ }^{4}$, Guri Grimnes ${ }^{2,5}$, Christopher Sivert Nielsen ${ }^{6,7}$, \\ Johanna U E Sollid ${ }^{8}$, Gunnar Skov Simonsen ${ }^{4,8}$, Bjørg Almås9 and Anne-Sofie Furberg 1,4,10
}

${ }^{1}$ Department of Community Medicine, Faculty of Health Sciences, UiT The Arctic University of Norway, Tromsø, Norway, ${ }^{2}$ Division of Internal Medicine, University Hospital of North Norway, Tromsø, Norway, ${ }^{3}$ Department of Pharmacy, Faculty of Health Sciences, UiT The Arctic University of Norway, Tromsø, Norway, ${ }^{4}$ Division of Internal Medicine, Department of Microbiology and Infection Control, University Hospital of North Norway, Tromsø, Norway, ${ }^{5}$ Endocrinology Research Group, Department of Clinical Medicine, Faculty of Health Sciences, UiT The Arctic University of Norway, Tromsø, Norway, ${ }^{6}$ Division of Chronic Diseases and Ageing, Norwegian Institute of Public Health, Oslo, Norway, ${ }^{7}$ Division of Emergencies and Critical Care, Department of Pain Management and Research, Oslo University Hospital, Oslo, Norway, ${ }^{8}$ Research Group for Host-Microbe Interaction, Department of Medical Biology, Faculty of Health Sciences, UiT The Arctic University of Norway, Tromsø, Norway, ${ }^{9}$ Hormone Laboratory, Department of Medical Biochemistry and Pharmacology, Haukeland University Hospital, Bergen, Norway, and ${ }^{10}$ Faculty of Health and Social Sciences, Molde University College, Molde, Norway

Correspondence should be addressed to D B Stensen Email dina.b.stensen@uit.no

\begin{abstract}
Objective: Staphylococcus aureus is a major human pathogen, and nasal carriers have an increased risk for infection and disease. The exploration of host determinants for nasal carriage is relevant to decrease infection burden. Former studies demonstrate lower carriage prevalence in women and among users of progestin-only contraceptives. The aim of this study was to investigate the possible associations between circulating sex-steroid hormones and nasal carriage of Staphylococcus aureus in a general population.

Methods: In the population-based sixth Tromsø study (2007-2008) nurses collected nasal swab samples from 724 women aged 30-87 not using any exogenous hormones, and 700 of the women had a repeated nasal swab taken (median interval 28 days). We analysed a panel of serum sex-steroids by liquid chromatography tandem mass spectrometry, and collected information about lifestyle, health and anthropometric measures. Multivariable logistic regression was used to study the association between circulating sex-steroids and Staphylococcus aureus carriage (one swab) and persistent carriage (two swabs), while adjusting for potential confounding factors. Women in luteal phase were excluded in the analysis of androgens.

Results: Staphylococcus aureus persistent nasal carriage prevalence was $22 \%$. One standard deviation increase in testosterone and bioavailable testosterone was associated with lower odds of persistent nasal carriage, $(\mathrm{OR}=0.57$; $95 \% \mathrm{Cl}=0.35-0.92$ and $\mathrm{OR}=0.52,95 \% \mathrm{Cl}=0.30-0.92)$ respectively. Analysis stratified by menopause gave similar findings. Persistent carriers had lower average levels of androstenedione and DHEA, however, not statistically significant.

Conclusion: This large population-based study supports that women with lower levels of circulating testosterone may have increased probability of Staphylococcus aureus persistent carriage.
\end{abstract}

(c) 2021 The authors Published by Bioscientifica Ltd. Printed in Great Britain 


\section{Introduction}

Staphylococcus aureus is the leading cause of skin and soft tissue infections, and can invade and infect any organ of the human body. $S$. aureus is the second most common cause of bloodstream infections (1), which continue to have high mortality among patients worldwide $(2,3)$. The burden of disease and mortality reflect $S$. aureus' extraordinary virulence factors, including highly evolved immune evasion strategies and resistance to antibiotic treatment (4).

S. aureus is frequent in the normal bacterial flora of healthy humans, and $20-50 \%$ of the population are nasal carriers $(4,5,6,7)$. Colonisation of the skin in the anterior nares is a major source of endogenous $S$. aureus infections and transmission (4). This motivates the search for lifestyle and environmental factors and associated biomarkers that may regulate immune responses to $S$. aureus. Among populations from different continents, male sex, younger age, atopic eczema, excess body weight, higher circulating glucose levels and diabetes, lower vitamin D levels, and work in health care services have been associated with higher probability of $S$. aureus nasal carriage, while smoking has been associated with both lower and higher probability of carriage $(4,5,8,9,10,11,12,13)$.

Sex-steroids are produced from cholesterol in testes or ovaries and the adrenal glands, and may be further converted to more potent sex-steroids in fat and other tissue. Sex-differences in immunological responses are largely caused by sex-steroid hormone actions, through binding to nuclear receptors and regulation of immune system genes expression in a variety of cells (14). In general, adult women have a more responsive immune system with faster clearance of pathogens and greater vaccine efficacy compared to men but are more prone to inflammatory and autoimmune diseases. Sex and age are the strongest risk factors for $S$. aureus nasal carriage $(4,8,9)$. Thus, it has been hypothesised that sex-steroid hormones are important in the immune response to $S$. aureus. Smaller studies among women have found a positive association of nasal S. aureus carriage with biomarkers of endogenous oestrogen level (15), as well as of nasal $S$. aureus carriage with exogenous oestrogen and progesterone (i.e. hormonal contraceptive combination preparations) $(16,17)$. One small study among premenopausal women found higher prevalence of persistent throat carriage of $S$. aureus with increasing levels of free fraction testosterone (18). From the population-based sixth Tromsø study (2007-2008), we reported higher nasal carriage among men and sexdifferences in the distribution of $S$. aureus spa types (genetic variance by S. aureus protein A (spa) typing) (9). Among 400 young women in the Tromsø study fit futures (2012-2013), where 50\% used hormonal contraception, we found a positive dose-response relationship between oestrogen dose and $S$. aureus nasal carriage, and a negative association between exogenous progesterone alone and nasal carriage (17). To our knowledge, no epidemiological study has examined whether endogenous sex-hormone levels are associated with $S$. aureus nasal carriage among women. Thus, the aim of this study is to investigate the possible associations between circulating sex-steroid hormones and nasal carriage of $S$. aureus in a general female population.

\section{Methods}

\section{Population and study design}

The Tromsø study includes seven extensive healthscreening surveys in the Tromsø municipality, North Norway, during 1974-2016 with invitation of total birth cohorts and large random samples of the population (5, 11). Each of these surveys consists of clinical examinations including anthropometric measures, non-fasting blood samples, interview covering menstrual history and hormonal medication, questionnaires on reproductive history, lifestyle, health and chronic disease $(5,11,12)$. The present study uses data from the sixth Tromsø study (the sixth Tromsø study, participation proportion 66\%).

Body height in centimetres $(\mathrm{cm})$ and weight in kilograms $(\mathrm{kg})$ were measured to the nearest 0.1 unit with participants wearing light clothing and no shoes. BMI was calculated as weight divided by height squared $\left(\mathrm{kg} / \mathrm{m}^{2}\right)$. Non-fasting blood samples were drawn from an antecubital vein. Glycated haemoglobin (HbA1c) was determined in EDTA-blood by HPLC using an automated analyser (Variant II, Bio-Rad Laboratories Inc.). The total analytical coefficient of variation was $<3.0 \%$. Serum 25-hydroxyvitamin D (25(OH)D) was analysed by electrochemiluminescence immunoassay (ECLIA) using an automated clinical chemistry analyser (Modular E170, Roche Diagnostics). The total analytical coefficient of variation was $7.3 \%$. There is a known overestimation of 25(OH)D levels in smokers when using the ECLIA (Roche) method (19). This necessitates stratification by smoking in the statistical analysis of $25(\mathrm{OH}) \mathrm{D}$.

Nasal swabs for $S$. aureus culturing were collected from a random sample of 4026 participants attending the sixth Tromsø study screening centre during October 2007 to June 2008, 2285 of these were women. All women 
without data on serum hormones, participants reporting antibiotic use or missing data on antibiotic use the last 24 $\mathrm{h}$, ongoing pregnancy, use of hormonal contraceptives, use of hormonal replacement therapy, endocrine breast cancer therapy and IVF treatment were excluded from the analysis. The participants were invited to a second visit for repeated nasal swab sampling. Women with invalid or missing $S$. aureus culturing results were excluded from the analysis (Fig. 1).

\section{Assessment of $S$. aureus carriage and serum sex-steroids}

Nurses at the sixth Tromsø study screening centre collected nasal swab samples. Each nasal vestibule was sampled with the same $\mathrm{NaCl}(0.9 \%)$-moistened sterile rayon-tipped swab which was rotated three times. The swabs were immediately placed in transport medium (Amies Copan, Brescia, Italy) and stored at $4^{\circ} \mathrm{C}$ for a maximum of 3 days. Trained personnel at the Department of Microbiology and Infection Control, University Hospital of North Norway, (UNN) Tromsø analysed the microbiological samples. The specimens were cultured on blood agar (Oxoid, UK) for growth control and chromID-plates (SAID) for $S$. aureus detection (bioMérieux, Marcy I'Etoile, France). The agar plates were incubated for $42-48 \mathrm{~h}$ at $37^{\circ} \mathrm{C}$. To retain high specificity, colony morphology was examined, and the most dominating colony type on the SAID plate was confirmed as $S$. aureus by the Staphaurex plus agglutination

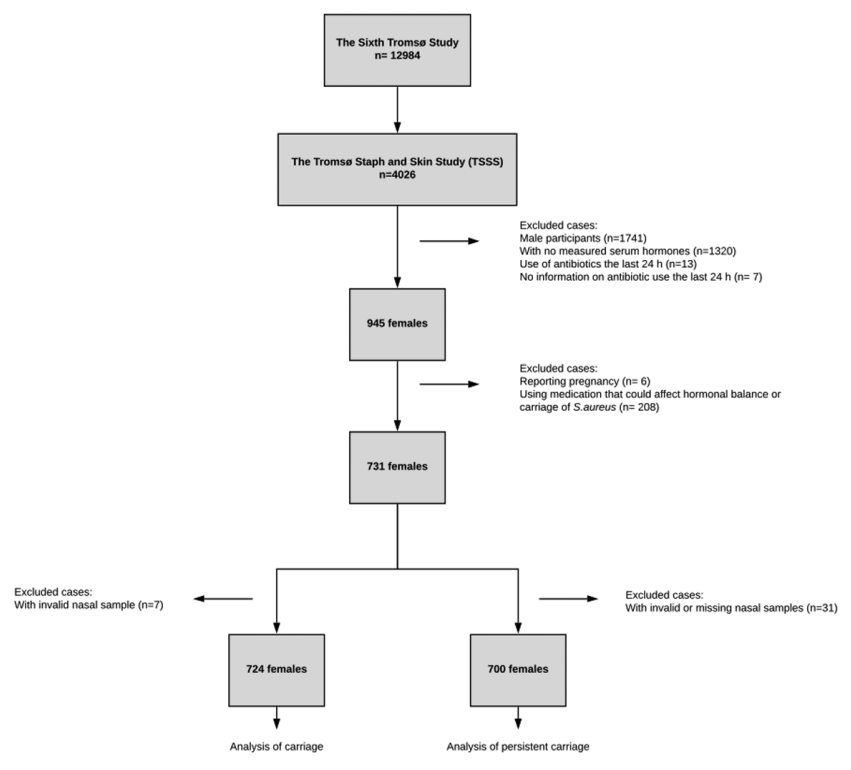

\section{Figure 1}

The study population. The sixth Tromsø study. test (Murex Diagnostic Ltd, Dartford, UK). Growth of any bacterial colonies on agar plates was registered as a valid culture.

The median interval between the first and the second nasal swab culture was 28 days.

S. aureus nasal carriage status was classified according to baseline sample status. $S$. aureus persistent carriage was defined as having two positive samples on the basis of van Belkum's definition of persistent carriage (20).

The Hormone laboratory at Haukeland University Hospital analysed a panel of serum sex-steroids by liquid chromatography tandem mass spectrometry (LCMS/MS, SCIEX API 5500 triple-quadrupole mass spectrometer, Applied Biosystems/MDS with an Agilent 1290 UPLC system), as part of the laboratory's work on establishing reference values within subgroups of the general population (i.e. by sex, age, and BMI categories) (21). LCMS/MS is the gold standard method for steroid profiling due to very high sensitivity and specificity (22). Serum concentrations of testosterone, androstenedione, dehydroandrostenedione (DHEAS), $17 \alpha$-hydroxyprogesterone (17-OH progesterone), progesterone, luteinising hormone (LH), folliclestimulating hormone (FSH), as well as the binding proteins sex-hormone binding globulin (SHBG) and albumin were measured using DPC immulite 200 XPi (Siemens Healthcare Diagnostics). For the estimation of bioavailable testosterone which includes free and albumin-bound testosterone we used the equation '(testosterone/SHBG) $\times 10^{\prime}(23)$ and the equation derived by Morris et al. (24).

\section{Classification of menopause and menstrual phase}

To classify menopausal status, we used the following question from the interview: 'Do you still have natural menstruation?' (yes/no/irregular/unknown status). We also defined FSH-level over 21.7 IU as postmenopausal and FSH-level under 21.7 IU as premenopausal $(25,26)$. Female participants reporting no menses with FSH-levels under 21.7 IU were further classified by the question from the interview: 'If you do not have natural menstruation, why did it stop?' (stopped by itself/I had a hysterectomy/ both ovaries were removed/other reasons (for instance radiation, cytotoxic mediation)). FSH-levels can be difficult to interpret because of the possible high levels in perimenopause, we therefore emphasised self-reported menstruation in classification of menopause. FSH-levels were used to confirm clinical history and to classify females with no data on self-reported menses. Among women with unknown status on menstruation from 
the interview, age was used as an additional criterium in those with FSH-levels above 21.7 IU (age $\geq 51$ years were classified as postmenopausal). One female participant reported no menses but had information about recent menstruation, we therefore concluded that this probably were due to misreporting on menstruation and classified the participant by FSH levels (Fig. 2).

Premenopausal women were further classified as follicular phase or luteal phase according to progesterone levels. Follicular phase were defined as progesterone levels

\section{Do you have menstruation?}

\section{Hysterectomy $(n=6)$}

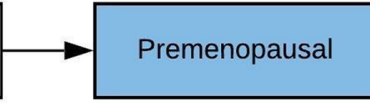

Stopped by itself $(n=2)$

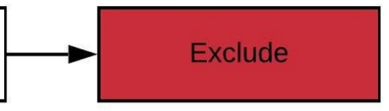

Information about last menstruation $(n=1)$
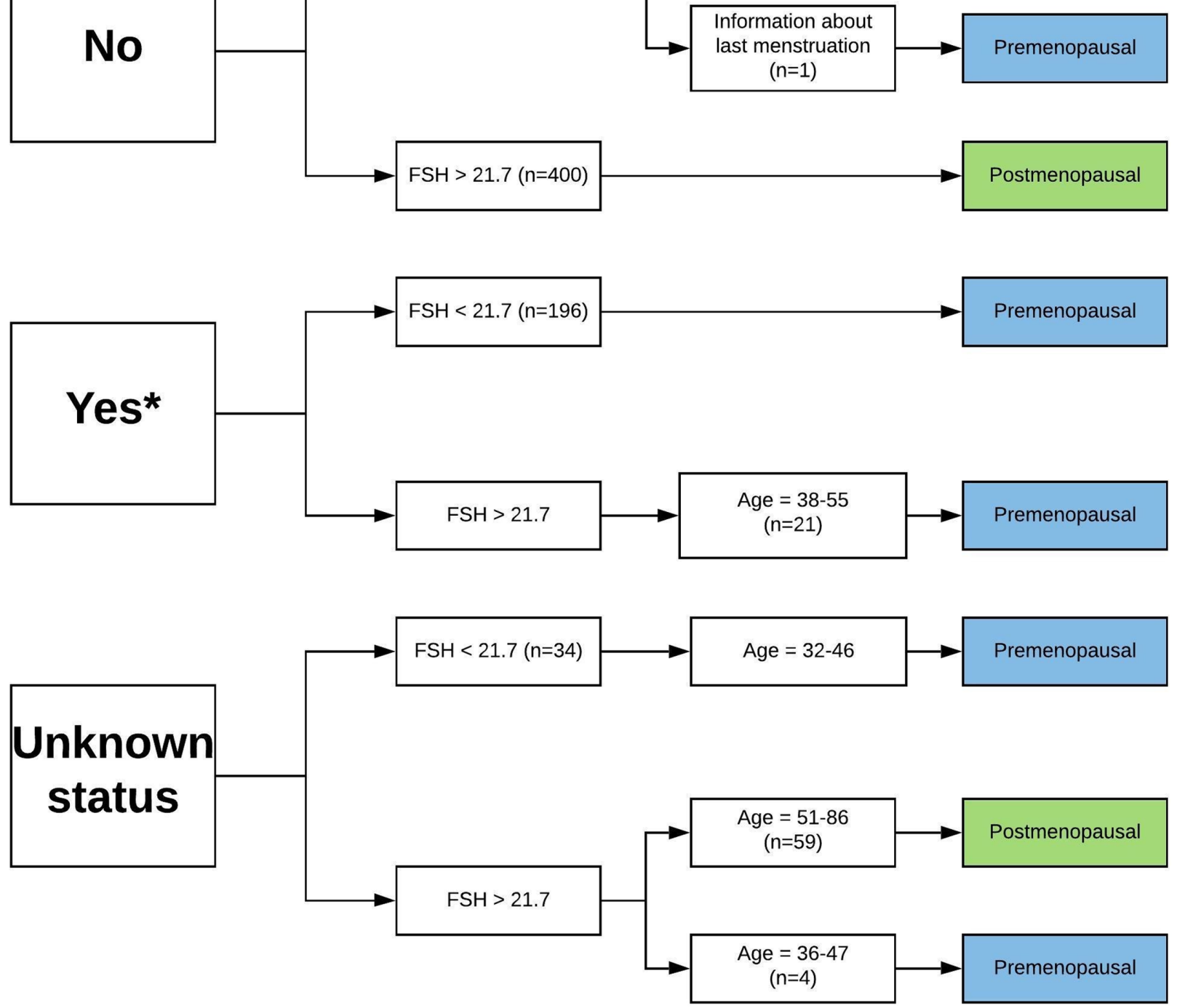

\section{Figure 2}

Classification of menstruation status, the sixth Tromsø study. 'Do you have menstruation?' corresponds with the interview question 'Do you still have natural menstruation?' *Participants reporting irregular menstruation $(n=2)$ are reclassified as 'yes'. FSH in IU, age in years. A full colour version of this figure is available at https://doi.org/10.1530/EJE-20-0877. 
$\leq 5.5 \mathrm{nmol} / \mathrm{L}$ and luteal phase as progesterone levels $>5.5$ $\mathrm{nmol} / \mathrm{L}$ (27). In the analysis including premenopausal women, women in the luteal phase are excluded to increase accuracy of the testosterone measurement (28).

\section{Statistical analysis}

Statistical analysis was performed using Stata/MP 15.1 for Macintosh. Univariable associations were analysed in contingency tables by calculating means and s.D. using chisquare and t-test to quantify the potential role of chance. Independent samples t-test were used to test possible associations between sex-steroid hormones, carriage and persistent carriage. Multivariable logistic regression was fitted to estimate odds ratios (ORs) and 95\% CIs to describe the associations between sex-steroid hormones and $S$. aureus nasal carriage and $S$. aureus persistent nasal carriage while adjusting for potential confounders. Missing values were excluded from the logistic regression analysis. DAGitty 3.0 was used for model selection (29). Testing for potential interaction between explanatory variables was done by including the multiplicative terms of two predictor variables in the model. $P$-values of $\leq 0.05$ were considered statistically significant.

\section{Ethics}

Each participant in the sixth Tromsø study signed a declaration of consent. The study does not include data from participants with their declaration of consent withdrawn after participation. Tromsø 6 was approved by the Regional Committee for Medical and Health Research Ethics (REK) and the Norwegian Data Protection Authority. The present analysis was approved by REK (2018/1975/REK nord).

\section{Results}

In this population of female participants with age 30-87 years $($ mean $=53.5$, S.D. $=13.6)$, the prevalence of $S$. aureus nasal carriage was $24 \%(180 / 724)$, and of $S$. aureus persistent nasal carriage 22\% (151/700) (Table 1 and Supplementary Table 1 , see section on supplementary materials given at the end of this article).

In postmenopausal women, mean level of testosterone were significantly lower in persistent nasal carriers compared to others (mean difference $(\mathrm{MD})=0.1095 \%$ $\mathrm{CI}=0.00-0.20$ ) (Table 2). When postmenopausal women's carrier state was assessed from only one nasal swab, the mean level of bioavailable testosterone was $0.15 \mathrm{nmol} / \mathrm{L}$ for non-carriers and $0.13 \mathrm{nmol} / \mathrm{L}$ for carriers $(\mathrm{MD}=0.02$ $95 \% \mathrm{CI}=-0.00$ to 0.04 ) (Supplementary Table 2). In postmenopausal women, serum levels of androstenedione, DHEAS, and $17 \alpha$-hydroxyprogesterone were lower in nasal carriers than in others but not statistically significant (Table 2).

Using DAGitty (29), age and BMI were identified as sufficient covariates in the regression analysis when estimating the association between sex-steroids and $S$. aureus nasal carriage in women. In a multivariable logistic regression model adjusted for age and BMI, an increase of 1 s.D. (s.D. $=0.81 \mathrm{nmol} / \mathrm{L}$ ) of testosterone lowered the odds of nasal carriage of $S$. aureus $(\mathrm{OR}=0.60 ; 95 \% \mathrm{CI}=0.39$ $0.94)$ as well as persistent nasal carriage $(\mathrm{OR}=0.57 ; 95 \%$ $\mathrm{CI}=0.35-0.92 ;$ S.D. $=0.82 \mathrm{nmol} / \mathrm{L})($ Table 3$)$.

When stratifying by menopausal status, this decrease in odds of persistent nasal carriage by higher testosterone was also found in postmenopausal women $(\mathrm{OR}=0.74 ; 95 \% \mathrm{CI}=0.55-0.99 ;$ s.D. $=0.43 \mathrm{nmol} / \mathrm{L})$. In premenopausal women, the association was similar but not statistically significant $(\mathrm{OR}=0.35 ; 95 \% \mathrm{CI}=0.05-2.29$; S.D. $=1.48 \mathrm{nmol} / \mathrm{L}$ ). The odds of nasal carriage by higher

Table 1 Characteristics of the study population by Staphylococcus aureus nasal carrier state. The sixth Tromsø study. Data are presented as mean \pm S.D. or as $n(\%)$.

\begin{tabular}{|c|c|c|c|c|c|c|}
\hline & \multicolumn{3}{|c|}{ Carrier state, $n=724$} & \multicolumn{3}{|c|}{ Persistent carrier state, $n=700$} \\
\hline & Non-carrier & Carrier & $P$-value ${ }^{a}$ & Others $^{\mathrm{b}}$ & Persistent carrier & $P$-value ${ }^{a}$ \\
\hline$n$ & 544 & 180 & & 549 & 151 & \\
\hline Age, years & $55.9 \pm 13.4$ & $55.1 \pm 13.7$ & 0.485 & $56.1 \pm 13.3$ & $56.7 \pm 13.3$ & 0.628 \\
\hline $\mathrm{BMI}, \mathrm{kg} / \mathrm{m}^{2}$ & $27.6 \pm 5.2$ & $28.2 \pm 5.6$ & 0.204 & $27.5 \pm 5.1$ & $28.4 \pm 5.8$ & 0.069 \\
\hline \multicolumn{7}{|l|}{ Menstruation phase } \\
\hline Menopause & $346(63.6 \%)$ & $113(62.8 \%)$ & 0.935 & 356 (64.9\%) & $103(68.2 \%)$ & 0.487 \\
\hline Luteal phase & $87(16.0 \%)$ & $28(15.5 \%)$ & & $87(15.8 \%)$ & $18(11.9 \%)$ & \\
\hline Follicular phase & $111(20.4 \%)$ & 39 (21.7 \%) & & $106(19.3 \%)$ & 30 (19.9 \%) & \\
\hline
\end{tabular}

${ }^{*}$ Chi-square test for categorical and t-tests for continuous variables; ${ }^{b}$ others; Intermittent carriers (one positive nasal samples of two samples in total) $n=$ 49; non-carriers (two negative nasal samples of two samples in total) $n=500$. 
Table 2 Mean difference and $\mathrm{Cl}$ in circulating sex-steroids, gonadotropins and binding protein levels among Staphylococcus aureus persistent nasal carriers compared to others. The sixth Tromsø study.

\begin{tabular}{|c|c|c|c|c|}
\hline & \multicolumn{2}{|c|}{ Premenopausal, $n=237^{\mathrm{a}, \mathrm{b}}$} & \multicolumn{2}{|c|}{ Postmenopausal, $n=445^{\mathrm{a}}$} \\
\hline & Mean difference $^{c}$ & $95 \% \mathrm{Cl}^{\mathrm{d}}$ & Mean difference $^{c}$ & $95 \% \mathrm{Cl}^{\mathrm{d}}$ \\
\hline Testosterone, nmol/L & 0.22 & -0.38 to 0.83 & 0.10 & $0.00-0.20$ \\
\hline Bioavailable testosterone ${ }^{\mathrm{e}}, \mathrm{nmol} / \mathrm{L}$ & 0.04 & -0.11 to 0.18 & 0.18 & -0.00 to 0.04 \\
\hline Androstenedione, $\mathrm{nmol} / \mathrm{L}$ & 0.17 & -0.42 to 0.76 & 0.12 & -0.13 to 0.36 \\
\hline Dehydroepiandrosterone, $\mathrm{nmol} / \mathrm{L}$ & -0.04 & -0.93 to 0.85 & 0.33 & -0.04 to 0.69 \\
\hline $17 \alpha$-hydroxyprogesterone, $\mathrm{nmol} / \mathrm{L}$ & 0.88 & -2.44 to 4.20 & 0.14 & -0.18 to 0.46 \\
\hline Progesterone, $\mathrm{nmol} / \mathrm{L}$ & 1.57 & -3.54 to 6.69 & 0.04 & -0.04 to 0.11 \\
\hline Sex-hormone binding globulin, $\mathrm{nmol} / \mathrm{L}$ & 9.91 & 0.73-19.09 & -0.89 & -7.72 to 5.93 \\
\hline Albumin, $\mathrm{nmol} / \mathrm{L}$ & 0.05 & -0.83 to 0.93 & 0.18 & -0.42 to 0.78 \\
\hline Luteinising hormone, IU & -0.26 & -2.99 to 2.47 & 0.80 & -1.67 to 3.26 \\
\hline Follicle-stimulating hormone, IU & 2.68 & -1.65 to 7.01 & 1.39 & -4.43 to 7.21 \\
\hline
\end{tabular}

aNumber may vary due to missing values; ${ }^{b}$ women in luteal phase are excluded in the analysis of testosterone, bioavailable testosterone, androstenedione and DHEA; ${ }^{c}$ mean difference $=$ mean (others) - mean (persistent carriage); ${ }^{\mathrm{d}}$ independent sample t-test; ebioavailable testosterone calculated from the equation '(testosterone/SHBG) $\times 10$ '.

testosterone were lower but not statistically significant for premenopausal women $(\mathrm{OR}=0.30 ; 95 \% \mathrm{CI}=0.06-$ 1.56; S.D. $=1.41 \mathrm{nmol} / \mathrm{L})$ and for postmenopausal women $(\mathrm{OR}=0.78 ; 95 \% \mathrm{CI}=0.59-1.02$; s.D. $=0.43 \mathrm{nmol} / \mathrm{L})$.

Multivariable logistic regression analysis showed a lower odds of nasal carriage $(\mathrm{OR}=0.53$; 95\% $\mathrm{CI}=0.32-$ $0.90)$ and persistent nasal carriage (OR $=0.52 ; 95 \%$ $\mathrm{CI}=0.30-0.92)$ for an increase in bioavailable testosterone by one standard deviation (s.D. $=0.19 \mathrm{nmol} / \mathrm{L}$ ) $($ Table 3$)$.

As for testosterone a decrease in odds of persistent nasal carriage was also found for postmenopausal women $(\mathrm{OR}=0.72 ; 95 \% \mathrm{CI}=0.52-0.99 ;$ s.D. $=0.09 \mathrm{nmol} / \mathrm{L})$. In premenopausal women, there was a similar association

Table 3 Associations between testosterone, bioavailable testosterone and Staphylococcus aureus nasal carriage and persistent carriage. Analysis on menopausal women and premenopausal women in follicular phase. Odds ratios (ORs) and 95\% Cls from logistic regression analysis. The sixth Tromsø study.

\begin{tabular}{|c|c|c|}
\hline & Nasal carriage & $\begin{array}{l}\text { Persistent nasal } \\
\text { carriage }\end{array}$ \\
\hline \multicolumn{3}{|l|}{ Testosterone } \\
\hline$n$ & 573 & 560 \\
\hline per S.D. ${ }^{a}$ & $0.60(0.39-0.94)$ & $0.57(0.35-0.92)$ \\
\hline $\mathrm{BMI}, \mathrm{kg} / \mathrm{m}^{2}$ & $1.03(0.99-1.07)$ & $1.03(0.99-1.07)$ \\
\hline Age, year & $0.99(0.98-1.01)$ & $1.00(0.98-1.02)$ \\
\hline \multicolumn{3}{|c|}{ Bioavailable testosterone } \\
\hline$n$ & 557 & 544 \\
\hline per S.D. ${ }^{b, c}$ & $0.53(0.32-0.90)$ & $0.52(0.30-0.92)$ \\
\hline $\mathrm{BMI}, \mathrm{kg} / \mathrm{m}^{2}$ & $1.05(1.01-1.09)$ & $1.06(1.01-1.10)$ \\
\hline Age, year & $0.99(0.98-1.01)$ & $1.00(0.98-1.01)$ \\
\hline
\end{tabular}

aSerum testosterone ( $\mathrm{nmol} / \mathrm{L}$ ) S.D.: analysis of nasal carriage, S.D. $=0.81$; analysis of persistent nasal carriage, S.D. $=0.82$; bioavailable testosterone $(\mathrm{nmol} / \mathrm{L}$ ) S.D.: analysis of nasal carriage, S.D. $=0.19$; analysis of persistent nasal carriage, S.D. $=0.19$; cioavailable testosterone calculated from the equation: (testosterone/SHBG) $\times 10$.
$(\mathrm{OR}=0.38 ; 95 \% \mathrm{CI}=0.06-2.45 ;$ s.D. $=0.34 \mathrm{nmol} / \mathrm{L})$. The odds of nasal carriage were lower but not statistically significant for premenopausal women $(\mathrm{OR}=0.28 ; 95 \%$ $\mathrm{CI}=0.05-1.46$; S.D. $=0.34 \mathrm{nmol} / \mathrm{L})$ and for postmenopausal women $(\mathrm{OR}=0.75 ; 95 \% \mathrm{CI}=0.56-1.01$; s.D. $=0.09 \mathrm{nmol} / \mathrm{L})$.

There was an inverse dose-response relationship between bioavailable testosterone and $S$. aureus carriage and persistent carriage, as well as for testosterone. In this female population, $98 \%$ had serum testosterone levels between 0 and $2 \mathrm{nmol} / \mathrm{L}$, the estimates for values over 2 $\mathrm{nmol} / \mathrm{L}$ are therefore more uncertain (Fig. 3).

Interaction analysis between all variables in the final model revealed no significant interactions. When the equation by Morris et al. was used to calculate bioavailable testosterone there was no change in the risk estimates for S. aureus carriage (Supplementary Table 5) (24). One female participant had an extreme value of testosterone (17.42 nmol/L, population-mean: $0.8 \mathrm{nmol} / \mathrm{L})$. Estimates from the final model did not differ when including or excluding this observation, and the presented results therefore include this observation.

\section{Discussion}

In this large population-based study of women not using any exogenous hormones, we report novel evidence of the association between circulating testosterone and bioavailable testosterone and $S$. aureus nasal carriage in women. This is to our knowledge the first study of circulating sex-steroids and $S$. aureus nasal carriage in a general female population. With increasing testosterone by one standard deviation, we see a $40 \%$ decrease in odds of $S$. aureus nasal carriage and a $43 \%$ decrease in $S$. aureus 

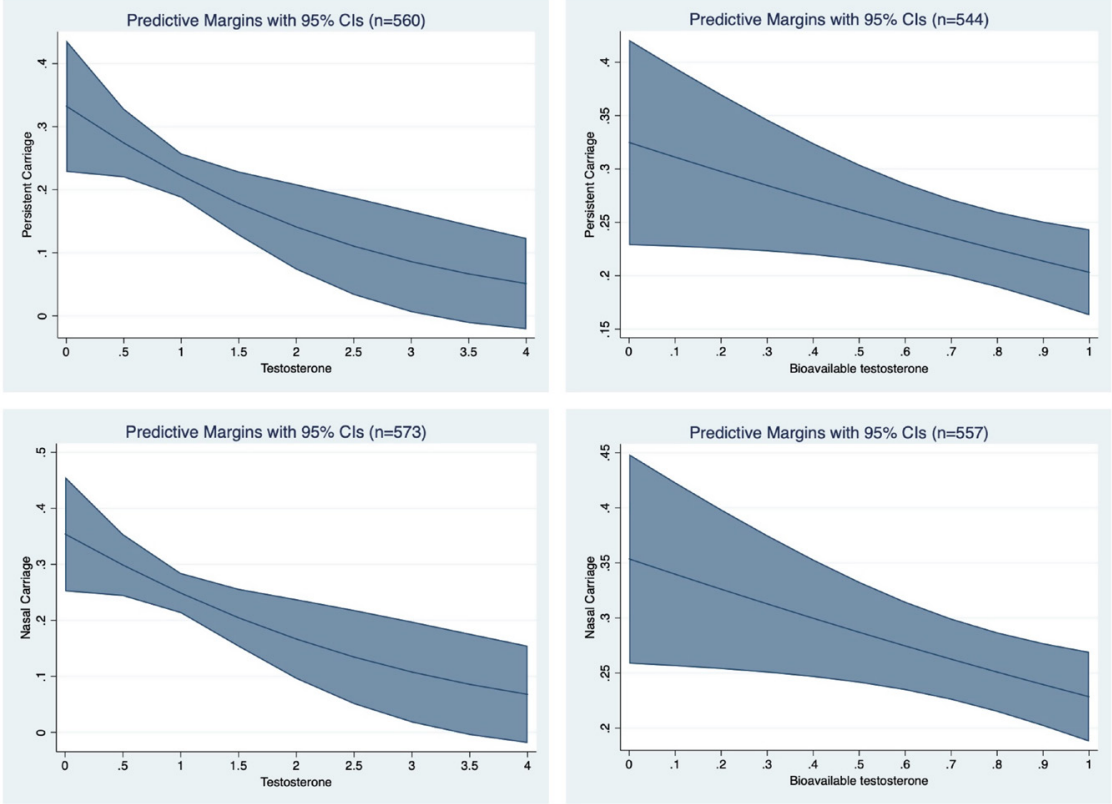

\section{Figure 3}

Probability of S. aureus nasal carriage (top) and persistent nasal carriage according to testosterone (left) and bioavailable testosterone (right). Analysis of postmenopausal women and premenopausal women in follicular phase. Testosterone and bioavailable testosterone per $\mathrm{nmol} / \mathrm{L}$. Bioavailable testosterone calculated from the equation '(testosterone/SHBG) $\times 10$ '. A full colour version of this figure is available at https:// doi.org/10.1530/EJE-20-0877. persistent nasal carriage compared to non-carriers and others (at least one of two nasal swab cultures negative for $S$. aureus), respectively. The same inverse associations are present in analysis stratified by menopausal status (range of ORs from 0.30 to 0.78 ), but statistically significant only for persistent nasal carriage in postmenopausal women.

We report a lowering in odds of $S$. aureus nasal carriage and $S$. aureus persistent nasal carriage of 47 and $48 \%$, respectively, with increase of bioavailable testosterone by one standard deviation compared to non-carriers and others. In postmenopausal women, the association with $S$. aureus persistent carriage was weaker, but statistically significant. All other estimates from analysis stratified by menopausal status show the same inverse associations between bioavailable testosterone and both nasal carriage and persistent nasal carriage, range of ORs from 0.28 to 0.75 , however, not statistically significant. Possibly, we lack the statistical power to identify a significant association because of the low number of participants in the subpopulations of premenopausal women $(n=135-$ 148). Persistent nasal carriers also had lower average levels of androstenedione and DHEAS, however not statistically significant.

The knowledge of host response during $S$. aureus nasal carriage is limited. The presence of $S$. aureus nasal carriage induces both innate and adaptive immune responses, but the bacteria overcome host defence mechanisms to establish carrier status $(6,30)$. Innate immunity exerts the first response against microbes, and the association between delayed response of toll- like receptor 2 in mice and nasal carriage has been demonstrated (31). In animal models, hypogonadism is associated with an increase in immune response with castration of male mice increasing autoimmune encephalomyelitis severity (32) and the incidence and severity of thyroiditis and adjuvant arthritis in rats (33). A recent study demonstrated an immunomodulating role of dihydrotestosterone in both human and rat vaginal smooth muscle cells (34). In summary, studies in animals document a significant effect of sex-steroid hormones on the immune system, but the results from in vitro studies are conflicting (35). Testosterone has been characterised as immunosuppressive, but recent research suggests that testosterones immunomodulatory effects depend on the reproductive context and menopausal status. There are also inconsistency of results for testosterone in both postmenopausal and premenopausal women where testosterone has been characterised as both proinflammatory and anti-inflammatory (36).

Former studies have shown a positive association between combination-hormonal contraceptives and $S$. aureus nasal carriage, but lower odds of carriage with progestin-only contraceptives. Studies have demonstrated lower levels of bioavailable testosterone in women using combined oral contraceptives (37). This supports the finding of lower odds of $S$. aureus nasal carriage in women with higher bioavailable testosterone. Progestin is a synthetic progestogen and is classified from its chemical structure as a derivate of testosterone or progesterone (38). Therefore, progestin has both androgen and progestogen activity when used in contraceptives, and progestin-only 
users may have a higher prevalence of androgenic side effects than combination-contraceptive users because of the lack of the oestrogen component (39).

High androgen and testosterone levels stimulate the sebaceous glands to increased sebum production, and plays an important role in the development of acne (40). In this study, $50-75 \%$ of women with polycystic ovary syndrome have high values of bioavailable testosterone and some of the diagnostic criteria are hyperandrogenistic features as acne (41). These hormonally related changes may also affect the microbiota of the skin and promote bacteria like Cutibacterium acnes that suppress the growth of other bacteria like $S$. aureus (42). This may be a plausible biological mechanism contributing to the lower probability of carriage of $S$. aureus with higher testosterone in our study.

Testosterone levels in women, as oestrogen levels, decline in the fourth decade of life and after menopause. Though the biological role of testosterone in women remains largely unclear, androgens are biological precursors of oestrogen production (43). High level of testosterone may therefore represent higher level of estrogen. However, oestrogen was not measured in our study. Estrogen has been theorised to have both proinflammatory and anti-inflammatory effects (44). One study found high testosterone and estradiol, and low SHBG levels associated with conditions that represent low-grade inflammation processes (45). The lower odds of S. aureus carriage with high levels of testosterone may be an indirect effect of possibly corresponding high levels of oestrogen or the unknown ratio between testosterone and oestrogen.

The major circulating steroids classified as androgens include DHEAS, androstenedione and testosterone (46). The lower levels of DHEAS and androstenedione in $S$. aureus persistent nasal carriers among postmenopausal women support our findings of lower odds of $S$. aureus carriage with higher levels of bioavailable testosterone and testosterone (Supplementary Fig. 2). This may represent an unknown effect of androgens on the immune system and increases the validity of the main finding.

We found no statistically significant association between androstenedione, LH, FSH, DHEAS, 17-OH progesterone, progesterone, SHBG, albumin and S. aureus nasal carriage and persistent carriage. This may be due to more complex relationships between sex-steroid hormones and the immune system. For premenopausal women, the female sex-steroid hormones have cyclic variations and data based on one measurement may not be representative for the participants' hormonal status.
It has been reported that the equation '(testosterone/ SHBG $\times 10)^{\prime}$ is not a reliable when SHBG concentration is low (47). Our analysis does not differ when using either this equation or the equation by Morris et al. (24) for the calculation of bioavailable testosterone. This may be due to our study population with mostly healthy women with normal BMI that has a low prevalence of very low measurements of SHBG $(48,49)$.

We used DAGitty for model selection, and the recommended confounders to adjust for in the analysis were BMI, HbA1c and age (Supplementary Fig. 1). Because of the minor insignificant effect on the main analysis of HbA1c, we decided to only adjust for BMI and age. We report a significant decrease of $S$. aureus with smoking, and a significant association with alcohol use. Because of the decision of not doing model selection by data driven statistical selection process, we did not include these variables in the final logistic regression model (Supplementary Tables 3 and 4). We include tables in Supplementary information for the reader to explore the odds ratio estimates including different possible covariates. The odds ratio estimates changed only slightly in these models. Although our data do not support any interactions among the explanatory variables, we cannot rule out the presence of smaller interaction effects that can only be detected by larger sample sizes.

Strengths of our study include a population-based design with high attendance, and consequently reduced risk of selection bias. Trained nurses collected nasal swab samples, and analyses of blood samples were performed with current gold standard assays for analysing sex-steroid hormones. Using both medical history and circulating FSH-levels to classify premenopausal and menopausal women reduces misclassification bias. One possible bias in the study is misclassification of $S$. aureus carrier status due to only two nasal swab samples, as van Belkum et al. recommend seven samples to correctly classify persistent carriers from intermittent carriers. Intermittent carriers has a lower risk of infections similar to non-carriers as well as similar elimination kinetics (20). Thus, we may have an unknown number of intermittent carriers misclassified as persistent in our study, which may have biased the risk estimates towards the null. However, large populationbased data reduces the risk of selection bias and increases both the internal and external validity.

In conclusion, we report evidence of an association between circulating testosterone and bioavailable testosterone and $S$. aureus nasal carriage defined from both one and two swab samples in females. Higher levels of bioavailable testosterone are associated with lower 
odds for S. aureus nasal carriage of 39-43\%. The role of testosterone and bioavailable testosterone in $S$. aureus nasal carriage ought to be addressed in future prospective studies to identify risk groups for prevention of $S$. aureus carriage and disease.

\section{Supplementary materials}

This is linked to the online version of the paper at https://doi.org/10.1530/ EJE-20-0877.

\section{Declaration of interest}

The authors declare that there is no conflict of interest that could be perceived as prejudicing the impartiality of this study.

\section{Funding}

This work was supported by the Northern Norway Regional Health Authority (grant number HNF1457-19) and the Odd Berg Group Medical Research fund 2007.

\section{Acknowledgements}

The authors thank each participant in the sixth Tromsø study for their collaboration, the staff at the screening center, the laboratory at the Department of Microbiology and Infection Control, University Hospital of North Norway, the laboratory at the Department of Medical Biochemistry University Hospital of North Norway and the hormone laboratory at Haukeland University Hospital. The authors also thank Bjørg C Haldorsen for technical assistance and Åshild Bjørnerem for excellent advice and for sharing her expert knowledge on sex-steroids.

\section{References}

1 NORM/NORM-VET. Usage of Antimicrobial Agents and Occurrence of Antimicrobial Resistance in Norway, 2015. Available from: https://ww w.vetinst.no/en/surveillance-programmes/norm-norm-vet-report

2 Tong SY, Davis JS, Eichenberger E, Holland TL \& Fowler Jr VG. Staphylococcus aureus infections: epidemiology, pathophysiology, clinical manifestations, and management. Clinical Microbiology Reviews 201528 603-661. (https://doi.org/10.1128/CMR.00134-14)

3 Paulsen J, Mehl A, Askim Å, Solligard E, Asvold BO \& Damas JK. Epidemiology and outcome of Staphylococcus aureus bloodstream infection and sepsis in a Norwegian county 1996-2011: an observational study. BMC Infectious Diseases 201515 116. (https:// doi.org/10.1186/s12879-015-0849-4)

4 Wertheim HF, Melles DC, Vos MC, van Leeuwen W, van Belkum A, Verbrugh HA \& Nouwen JL. The role of nasal carriage in Staphylococcus aureus infections. Lancet: Infectious Diseases 20055 751-762. (https://doi.org/10.1016/S1473-3099(05)70295-4)

5 Olsen K, Falch BM, Danielsen K, Johannessen M, Ericson Sollid JU, Thune I, Grimnes G, Jorde R, Simonsen GS \& Furberg AS. Staphylococcus aureus nasal carriage is associated with serum 25-hydroxyvitamin D levels, gender and smoking status. The Tromso Staph and Skin Study. European Journal of Clinical Microbiology and Infectious Diseases 201231 465-473. (https://doi.org/10.1007/s10096011-1331-x)

6 Sollid JU, Furberg AS, Hanssen AM \& Johannessen M. Staphylococcus aureus: determinants of human carriage. Infection, Genetics and Evolution 201421 531-541. (https://doi.org/10.1016/j. meegid.2013.03.020)

7 Verhoeven PO, Gagnaire J, Botelho-Nevers E, Grattard F, Carricajo A, Lucht F, Pozzetto B \& Berthelot P. Detection and clinical relevance of Staphylococcus aureus nasal carriage: an update. Expert Review of Anti-Infective Therapy 201412 75-89. (https://doi.org/10.1586/14787 210.2014.859985)

8 Johannessen M, Sollid JE \& Hanssen AM, Host. Host- and microbe determinants that may influence the success of $\mathrm{S}$. aureus colonization. Frontiers in Cellular and Infection Microbiology 2012256. (https://doi.org/10.3389/fcimb.2012.00056)

9 Sangvik M, Olsen RS, Olsen K, Simonsen GS, Furberg AS \& Sollid JU. Age- and gender-associated Staphylococcus aureus spa types found among nasal carriers in a general population: the Tromso Staph and Skin Study. Journal of Clinical Microbiology 201149 4213-4218. (https://doi.org/10.1128/JCM.05290-11)

10 Matheson EM, Mainous 3rd AG, Hueston WJ, Diaz VA \& Everett CJ. Vitamin D and methicillin-resistant Staphylococcus aureus nasal carriage. Scandinavian Journal of Infectious Diseases 201042 455-460. (https://doi.org/10.3109/00365541003602049)

11 Olsen K, Danielsen K, Wilsgaard T, Sangvik M, Sollid JU, Thune I, Eggen AE, Simonsen GS \& Furberg AS. Obesity and Staphylococcus aureus nasal colonization among women and men in a general population. PLoS ONE 20138 e63716. (https://doi.org/10.1371/ journal.pone.0063716)

12 Sorensen M, Wickman M, Sollid JU, Furberg AS \& Klingenberg C. Allergic disease and Staphylococcus aureus carriage in adolescents in the Arctic region of Norway. Pediatric Allergy and Immunology 201627 728-735. (https://doi.org/10.1111/pai.12595)

13 Durmaz R, Tekerekoglu MS, Kalcioglu T \& Ozturan O. Nasal carriage of methicillin-resistant Staphylococcus aureus among smokers and cigarette factory workers. New Microbiologica 2001 24 143-147.

14 Klein SL \& Flanagan KL. Sex differences in immune responses. Nature Reviews: Immunology 201616 626-638. (https://doi.org/10.1038/ nri.2016.90)

15 Winkler J, Block C, Leibovici L, Faktor J \& Pitlik SD. Nasal carriage of Staphylococcus aureus: correlation with hormonal status in women. Journal of Infectious Diseases 1990162 1400-1402. (https://doi. org/10.1093/infdis/162.6.1400)

16 Zanger P, Nurjadi D, Gaile M, Gabrysch S \& Kremsner PG. Hormonal contraceptive use and persistent Staphylococcus aureus nasal carriage. Clinical Infectious Diseases 201255 1625-1632. (https://doi. org/10.1093/cid/cis778)

17 Stensen DB, Smabrekke L, Olsen K, Grimnes G, Nielsen CS, Simonsen GS, Sollid JUE \& Furberg AS. Hormonal contraceptive use and Staphylococcus aureus nasal and throat carriage in a Norwegian youth population. PLOS ONE 201914 e0218511. (https://doi. org/10.1371/journal.pone.0218511)

18 Nowak JE, Borkowska BA \& Pawlowski BZ. Sex differences in the risk factors for Staphylococcus aureus throat carriage. American Journal of Infection Control 201745 29-33. (https://doi.org/10.1016/j. ajic.2016.07.013)

19 Grimnes G, Almaas B, Eggen AE, Emaus N, Figenschau Y, Hopstock LA, Hutchinson MS, Methlie P, Mihailova A, Sneve M et al. Effect of smoking on the serum levels of 25-hydroxyvitamin D depends on the assay employed. European Journal of Endocrinology 2010163 339-348. (https://doi.org/10.1530/EJE-10-0150)

20 van Belkum A, Verkaik NJ, de Vogel CP, Boelens HA, Verveer J, Nouwen JL, Verbrugh HA \& Wertheim HF. Reclassification of Staphylococcus aureus nasal carriage types. Journal of Infectious Diseases 2009199 1820-1826. (https://doi.org/10.1086/599119)

21 Methlie P, Hustad SS, Kellmann R, Almas B, Erichsen MM, Husebye E \& Lovas K. Multisteroid LC-MS/MS assay for glucocorticoids and androgens, and its application in Addison's disease. Endocrine Connections 20132 125-136. (https://doi.org/10.1530/EC-13-0023) 
22 Hsing AW, Stanczyk FZ, Belanger A, Schroeder P, Chang L, Falk RT \& Fears TR. Reproducibility of serum sex steroid assays in men by RIA and mass spectrometry. Cancer Epidemiology, Biomarkers and Prevention 200716 1004-1008. (https://doi.org/10.1158/1055-9965. EPI-06-0792)

23 Channer KS \& Jones TH. Cardiovascular effects of testosterone: implications of the 'male menopause'? Heart 200389 121-122. (https://doi.org/10.1136/heart.89.2.121)

24 Morris PD, Malkin CJ, Channer KS \& Jones TH. A mathematical comparison of techniques to predict biologically available testosterone in a cohort of 1072 men. European Journal of Endocrinology 2004151 241-249. (https://doi.org/10.1530/ eje.0.1510241)

25 Mellgren G. FSH - Helse Bergen HF Haukeland Universitetssjukehus, 2019 [updated 28.02.19]. (available from: https://analyseoversikten . no/analyse/15?query=fsh)

26 Kühnel W. IMMULITE and IMMULITE 2000 Reference Range Compendium, 2000 [First English Edition]. (available at: http://www .dpcweb.com/documents/medical/reference_ranges/ZB197-A.pdf)

27 Mellgren MG. Progesteron, 2020. (available from: https://analyse oversikten.no/analyse/32)

28 Kanakis GA, Tsametis CP \& Goulis DG. Measuring testosterone in women and men. Maturitas 2019125 41-44. (https://doi. org/10.1016/j.maturitas.2019.04.203)

29 Textor J, van der Zander B, Gilthorpe MS, Liskiewicz M \& Ellison GT. Robust causal inference using directed acyclic graphs: the R package 'dagitty'. International Journal of Epidemiology 201645 1887-1894. (https://doi.org/10.1093/ije/dyw341)

30 Azziz R, Dumesic DA \& Goodarzi MO. Polycystic ovary syndrome: an ancient disorder? Fertility and Sterility 201195 1544-1548. (https:// doi.org/10.1016/j.fertnstert.2010.09.032)

31 Quinn GA \& Cole AM. Suppression of innate immunity by a nasal carriage strain of Staphylococcus aureus increases its colonization on nasal epithelium. Immunology 2007122 80-89. (https://doi. org/10.1111/j.1365-2567.2007.02615.x)

32 Bebo Jr BF, Zelinka-Vincent E, Adamus G, Amundson D, Vandenbark AA \& Offner H. Gonadal hormones influence the immune response to PLP 139-151 and the clinical course of relapsing experimental autoimmune encephalomyelitis. Journal of Neuroimmunology 199884 122-130. (https://doi.org/10.1016/s01655728(97)00214-2)

33 Ansar Ahmed S, Young PR \& Penhale WJ. Beneficial effect of testosterone in the treatment of chronic autoimmune thyroiditis in rats. Journal of Immunology $1986 \mathbf{1 3 6}$ 143-147.

34 Maseroli E, Cellai I, Filippi S, Comeglio P, Cipriani S, Rastrelli G, Rosi M, Sorbi F, Fambrini M, Petraglia F et al. Anti-inflammatory effects of androgens in the human vagina. Journal of Molecular Endocrinology 202065 109-124. (https://doi.org/10.1530/ JME-20-0147)
35 Bouman A, Heineman MJ \& Faas MM. Sex hormones and the immune response in humans. Human Reproduction Update 200511 411-423. (https://doi.org/10.1093/humupd/dmi008)

36 Lorenz TK, Heiman JR \& Demas GE. Testosterone and immunereproductive tradeoffs in healthy women. Hormones and Behavior 201788 122-130. (https://doi.org/10.1016/j.yhbeh.2016.11.009)

37 Zimmerman Y, Eijkemans MJ, Coelingh Bennink HJ, Blankenstein MA \& Fauser BC. The effect of combined oral contraception on testosterone levels in healthy women: a systematic review and meta-analysis. Human Reproduction Update 201420 76-105. (https://doi.org/10.1093/humupd/dmt038)

38 Sitruk-Ware R. Pharmacological profile of progestins. Maturitas 2004 47 277-283. (https://doi.org/10.1016/j.maturitas.2004.01.001)

39 Azziz R, Nestler JE \& Dewailly D. Androgen Excess Disorders in Women. New Jersey: Humana Press Inc., 2006.

40 Dreno B, Martin R, Moyal D, Henley JB, Khammari A \& Seite S. Skin microbiome and acne vulgaris: Staphylococcus, a new actor in acne. Experimental Dermatology 201726 798-803. (https://doi.org/10.1111/ exd.13296)

41 Goodarzi MO, Dumesic DA, Chazenbalk G \& Azziz R. Polycystic ovary syndrome: etiology, pathogenesis and diagnosis. Nature Reviews: Endocrinology 20117 219-231. (https://doi.org/10.1038/ nrendo.2010.217)

42 Grice EA \& Segre JA. The skin microbiome. Nature Reviews: Microbiology 20119 244-253. (https://doi.org/10.1038/nrmicro2537)

43 Horstman AM, Dillon EL, Urban RJ \& Sheffield-Moore M. The role of androgens and estrogens on healthy aging and longevity. Journals of Gerontology: Series A, Biological Sciences and Medical Sciences 201267 1140-1152. (https://doi.org/10.1093/gerona/gls068)

44 Straub RH. The complex role of estrogens in inflammation. Endocrine Reviews 200728 521-574. (https://doi.org/10.1210/er.2007-0001)

45 Liva SM \& Voskuhl RR. Testosterone acts directly on CD4+ T lymphocytes to increase IL-10 production. Journal of Immunology 2001 167 2060-2067. (https://doi.org/10.4049/jimmunol.167.4.2060)

46 Burger HG. Androgen production in women. Fertility and Sterility 200277 (Supplement 4) S3-S5. (https://doi.org/10.1016/s00150282(02)02985-0)

47 Keevil BG, Adaway J, Fiers T, Moghetti P \& Kaufman JM. The free androgen index is inaccurate in women when the SHBG concentration is low. Clinical Endocrinology 201888 706-710. (https://doi.org/10.1111/cen.13561)

48 Keevil BG \& Adaway J. Assessment of free testosterone concentration. Journal of Steroid Biochemistry and Molecular Biology 2019190 207-211. (https://doi.org/10.1016/j.jsbmb.2019.04.008)

49 Rosner W, Auchus RJ, Azziz R, Sluss PM \& Raff H. Position statement: utility, limitations, and pitfalls in measuring testosterone: an Endocrine Society position statement. Journal of Clinical Endocrinology and Metabolism 200792 405-413. (https://doi. org/10.1210/jc.2006-1864)

Received 1 August 2020

Revised version received 23 November 2020

Accepted 16 December 2020 